\section{Kongress der Deutschen Gesellschaft für Gynäkologie und Geburtshilfe (DGGG) in München (Juni 2000)}

\section{Bericht über die erste wissenschaftliche Sitzung nach Neugründung der Arbeitsgemeinschaft Balneologie, Physiotherapie, Rehabilitation und Akupunktur in der Frauenheilkunde e. V. in der DGGG}

Gynäkologische Bäderbehandlungen haben als Ergänzung zu operativen und medikamentösen Therapieformen eine lange Tradition.

Bereits 1980 wurde auf der Jahrestagung der DGGG eine Sektion «Balneogynäkologie» von $H$. Baatz gegründet.

Auf dem 52. Kongress in Nürnberg 1998 wählte die Arbeitsgemeinschaft Gynäkologische Balneologie, Physiotherapie und Rehabilitation A.-M. Beer (Hattingen) zum ersten Vorsitzenden, C. Karl (Eschweiler) zum zweiten Vorsitzenden und R. F. Baumbach (Bad Schwalbach) zum Schriftführer und Schatzmeister.

Bereits 1980 wird im Gründungsprotokoll der Sektion «Balneogynäkologie» von den damaligen Vorsitzenden Baatz und Beck beklagt, dass die gesamte jüngere Generation auf dem Gebiet der gynäkologischen Balneotherapie ein Defizit zu verzeichnen habe, da dieses Fachgebiet in der Lehre an den Universitäten kaum vertreten sei.

An dieser Situation hat sich bis heute leider, bis auf wenige Ansätze, keine spürbare Änderung ergeben. Daher wurde die Arbeitsgemeinschaft Gynäkologische Balneotherapie, Phytotherapie und Rehabilitation unter dem Namen «Arbeitsgemeinschaft Balneologie, Physiotherapie, Rehabilitation und Akupunktur in der Frauenheilkunde e. V.» als eingetragener Verein mit eigener Mitgliedschaft fortgeführt. Gleichzeitig wurden auch Frauenärzte, die Akupunktur anwenden, zur Mitgliedschaft eingeladen. So ist erstmals auch die Akupunktur in einer wissenschaftlichen Arbeitsgemeinschaft der DGGG vertreten. Der Vorstand der DGGG hat diese Tatsache begrüsst.

Die wissenschaftliche Sitzung der Arbeitsgemeinschaft auf dem 53. Kongress der DGGG war durch zwei Themenbereiche charakterisiert. Vier Vorträge fanden zum Thema Balneologie, Physiotherapie und Rehabilitation und 3 Vorträge zum Thema Akupunktur statt. Die wichtigsten Ergebnisse sollen im folgenden dargestellt werden.

C. Goecke (Aachen) sprach zum Thema «Gynäkologische Onkologie - Notwendigkeit der Rehabilitation aus der Sicht eines Akutkrankenhauses».

Bisher kämen nur weniger als 1\% der Rehabilitations-Patienten aus dem gynäkologischen Fachbereich. Dabei könnten Patientin- nen nach ausgedehnten gynäkologischen Karzinomoperationen (Mammakarzinom nach begonnener Chemotherapie und/oder Strahlentherapie) und nach Abschluss der Primärbehandlung in die Anschlussheilbehandlung (AHB) und die Rehabilitation geschickt werden. Geeignet sind Frauenheilbäder, die über die notwendige Struktur- und Prozessqualität zu Durchführungen gynäkologischer AHB und Rehabilitation verfügen.

In seinem Vortrag «Rehabilitation versus stationäre Naturheilkunde in der Frauenheilkunde» stellte A.-M. Beer (Hattingen) anhand von Datenmaterial, das im Rahmen der wissenschaftlichen Begleitung in der Modellabteilung für Naturheilkunde in HattingenBlankenstein erhoben wurde, fest, dass die Versorgung mit klassischen Naturheilverfahren im stationären Akutbereich eine effektive, wissenschaftlich anerkannte Alternative bzw. Ergänzung zum rehabilitativen Angebot im Bereich der Frauenheilkunde darstellt. Die hohe Therapiedichte und Patientenzufriedenheit liesse sich im stationär-naturheilkundlichen Bereich in der Frauenheilkunde ebenso erreichen wie in der Rehabilitation.

Torf und Torfprodukte werden indikationsbezogen erfolgreich in der Frauenheilkunde eingesetzt. Dabei galt bislang der physikalisch-thermische Effekt als Hauptwirkkomponente. Darüber hinaus hat es sich jedoch gezeigt, dass auch biochemische Wirkungen durch die Torfinhaltsstoffe vorhanden sind und für die Wirksamkeit des Torfes mitverantwortlich sind. Die beiden folgenden Vorträge beschäftigten sich mit dieser Fragestellung.

$U$. Riede (Freiburg i. Br.) sprach über die proinflammatorische Wirkung der Huminstoffe auf Epidermiszellen. Es habe sich gezeigt, dass Huminstoffe und Huminsäuren proinflammatorisch wirken. Dies konnte an menschlichen Keratinozyten der Epidermis in Kokulturen mit polymorphnukleären Neutrophilen nachgewiesen werden. Die Zerstörungen des Kultur-Monolayers durch Huminstoffe waren denen durch TNF- $\alpha$ vergleichbar.

$R$. Köcking (Jena) stellte «Untersuchungen zur antiviralen Aktivität von polyanionischen Torfinhaltstoffen in vitro und in vivo» vor. Sie schlussfolgerte, dass der prophylaktische Schutz von Haut und Schleimhaut mit polyanionischen Virusinhibitoren zu einem interessanten Anwendungsgebiet für bestimmte Torfinhaltsstoffe werden könnte.

Der zweite Teil der wissenschaftlichen Sitzung beinhaltete Vorträge zum Thema Akupunktur.

A. Römer (Mannheim) stellte eine Akupunktur-Studie zum Thema «Karpaltunnelsyndrom in der Schwangerschaft» vor. Dieses Krankheitsbild würde häufig unterschätzt und sei mit Akupunktur erfolgreich zu behandeln.

Bislang gibt es keine einheitlichen Vorgaben zur Ausbildung von Frauenärzten in der Akupunktur.

In den Curricula der verschiedenen Ausbildungsinstitute für Akupunktur bzw. Traditionelle Chinesische Medizin ist sie meist unter-

\section{KARGER}

Fax +497614520714

E-mail Information@Karger.de

www.karger.com (c) 2000 S. Karger GmbH, Freiburg

Accessible online at:

www.karger.com/journals/fkm
Dr. med. André-Michael Beer

Modellabteilung für Naturheilkunde

Klinik Blankenstein

Im Vogelsang 5-11, D-45527 Hattingen (Deutschland)

Tel. +49 232439 64-87, Fax -97

E-mail wellbeer@cityweb.de 
repräsentiert. Das Tang-Du-Institut, das Ausbildungsmöglichkeiten für Gynäkologie und Geburtshilfe anbietet, arbeitet seit 1998 mit dem Berufsverband der Frauenärzte zusammen.

Daher wurde J. Tang (Herdecke) gebeten einen Einblick in diese Ausbildungsform zu geben. Das grosse Interesse und der Ausbildungsbedarf werde durch eine hohe Zahl von Neuanmeldungen bestätigt. In den letzten 2 Jahren seien von ihrem Institut 197 Teilnehmer ausgebildet worden, wobei 122 mit der Abschlussprüfung abgeschlossen hätten.

Die Arbeitsgemeinschaft der wissenschaftlichen medizinischen Fachgesellschaften (AWMF) hat die angeschlossenen Fachgesellschaften um die Erarbeitung von Leitlinien zur Diagnostik und Therapie gebeten. Seitens der DGGG wurden daher bereits 1996 die Arbeitsgemeinschaften darum gebeten, zügig an die Erarbeitung der Leitlinien heranzugehen.

Im Frühjahr 2000 initiierte die neu gegründete Arbeitsgemeinschaft daher erstmals ein Treffen, zu dem die Arbeitsgemeinschaft Naturheilverfahren und Umweltmedizin und weitere Fachkollegen anderer Gesellschaften nach Hattingen-Blankenstein eingeladen wurden.
A.-M. Beer (Hattingen) zeigte Ziele und Inhalte der neu geformten Leitlinien zur Anwendung und Ausbildung der Akupunktur und ihrer verschiedenen Behandlungsformen in der Gynäkologie und Geburtshilfe auf.

Diese sollen dazu dienen, die Qualität der Akupunktur-Behandlung zu sichern, Ärzten und Patienten Sicherheit bei der Behandlung zu geben und den Berufsverbänden für die Gestaltung und Durchführung der Akupunkturausbildung Anhalte zu bieten. Eine 70-stündige fachspezifische Ausbildung (Gynäkologie und Geburtshilfe) würde für erforderlich erachtet. A.-M. Beer folgerte, dass zeitnah die vollständige Erarbeitung der Leitlinien anhand der von der AWFM vorgelegten Qualitätskriterien und Empfehlungen erfolgen sollte, und wies abschliessend darauf hin, dass die 2. Sitzung zur Erarbeitung der Leitlinien im Rahmen des Kongresses stattfinden werde und die Diskussion im August 2000 in Frankfurt/M. fortgesetzt würde. Interessenten, die Akupunktur in der Frauenheilkunde und Geburtshilfe anwenden, sind herzlich zur Mitarbeit aufgerufen.

A.-M. Beer, Hattingen

\section{Tagung Naturheilverfahren in Berchtesgaden KURS A}

\section{1. bis 5 . November 2000}

\section{Themenschwerpunkte}

Phytotherapie

(Wirksamkeit/Verschreibung, konservative/phytopharmakotherapeutische Möglichkeiten bei chronischer Insuffizienz, Erkältung, Infekt und Bronchitis)

\section{Bewegungstherapie}

(Bewegung und Sport, Grundlagen - Adaption - Ausdauerkrafttraining, Segmentmassagen, manuelle Lymphdrainage, bei Osteoporose, physikalische Therapie, manuelle Diagnostik, BG-Massagen und Periostbehandlung, Massagetherapie, Kolonbehandlung, Atemtherapie)

\section{Hydro-Thermotherapie}

(Wickel, Packungen, Teilbäder, Güsse, Waschungen, Kneipp-Therapie, Überwärmungsbäder, Kräuterbäder, pflanzliche Badezusätze/Wirkung, Sauna, Dampfbäder)

Symbioselenkung/Mikrobiologische Therapie

Klima-Lichttherapie

Balneo-Thalassotherapie

andere Therapieprinzipien

Für die Erlangung der Zusatzbezeichnung «Naturheilverfahren» muss ein gesamter Kursblock A-D besucht werden. Die Kursabfolge ist nicht vorgeschrieben, der «Neu-Einstieg» ist bei jedem Kurs möglich.

\begin{abstract}
Information und Anmeldung:
Berufsverband Deutscher Internisten e. V.

Schöne Aussicht 5, D-65193 Wiesbaden

Tel. +49 611181 33-22 oder -24, Fax -23

E-mailinfo@bdi.de,www.bdi.de
\end{abstract}

Teilnahmegebühr:

DM 450,- für Mitglieder des Berufsverbandes

DM 550,- für Nichtmitglieder 